\title{
Quo vadis Diabetologie? Weißbuch Diabetes deckt Mängel in der Versorgung auf
}

\author{
Das Weißbuch Diabetes, vorgestellt \\ auf der diesjährigen Jahrestagung \\ der Deutschen Diabetologischen \\ Gesellschaft in Stuttgart, liefert eine \\ aktuelle wissenschaftliche Bestands- \\ aufnahme über die Versorgung von \\ Patienten mit Diabetes in Deutsch- \\ land. Das Ergebnis: Es gibt reichlich \\ Optimierungsbedarf.
}

In den letzten Jahren konnte zwar einiges erreicht werden, etwa eine verbesserte Stoffwechseleinstellung. Dennoch: „Bisher erreichen noch nicht genug Patienten den Idealbereich zur Prävention von Folgeerkrankungen“, betonte Prof. Bertram Häussler vom IGES Institut, Berlin.

Gerade die Spätfolgen des Diabetes führen aber zu den wesentlichen Zusatzkosten, erklärte Prof. Thomas Danne, Vorstandsvorsitzender von DiabetesDE. Die Patienten müssen deshalb so früh und so gut wie möglich behandelt werden.
Angesichts einer stetig zunehmenden Diabetesprävalenz forderte er eine nationale Diabetesstrategie, wie sie bereits bei knapp der Hälfte der Länder in der Europäischen Gemeinschaft realisiert ist. Sie könne zur Koordination der Maßnahmen für eine bessere Prävention, eine bestmögliche Versorgung und die kontinuierliche Erforschung der Volkskrankheit führen. Da es sich beim Diabetes um ein multifaktorielles Geschehen handelt, bringe ein nicht abgestimmtes Verfahren wenig.

Zudem machte er die Notwendigkeit einer effektiven Primärprävention schon allein aus Kostengründen deutlich: „Nur, wenn es gelingt in der Primärprävention erfolgreich zu sein und die Zahl der Erkrankungen langfristig zu reduzieren, werden wir die Kosten für eine bestmögliche Versorgung der Erkrankten auf Dauer aufbringen können."

Im Fokus präventiver Strategien steht dabei der Risikofaktor Nummer 1: das Übergewicht. Bereits ab dem vierten Lebensjahr sollte aus Sicht von Danne mit der Primärprävention begonnen werden. DiabetesDE macht sich deshalb für eine Stunde Sport pro Tag in Schulen und Kindergärten, ein Schulfach "Gesundheit und Ernährung" und eine klare Lebensmittelkennzeichnung, etwa in Form einer Nährwertampel, stark.

Häussler mahnte zudem Änderungen in der Beurteilung innovativer Antidiabetika an. Aus seiner Sicht sollten ärztliche Erfahrungen aus der Routineversorgung, aber auch die Meinung der Patienten in die Bewertung einfließen. Noch würde die Patientenperspektive nicht ausreichend berücksichtigt. Dementsprechend empfiehlt das Weißbuch ein Stimmrecht von Patientenvertretern im Gemeinsamen Bundesausschuss (G-BA).

(bf) II

Pressekonferenz „Weißbuch Diabetes 2010 Bestandsaufnahme und Zukunftskonzepte für die Versorgung einer Volkskrankheit“", Stuttgart, 12. Mai 2010 (Veranstalter: Roche)

\section{Kardiorenales Anämiesyndrom Von Eisen profitieren Herz und Nieren}

\begin{abstract}
Patienten mit Herz- und Niereninsuffizienz leben besser und möglicherweise auch länger, wenn man ihre Eisenspeicher durch eine intravenöse Substitution auffüllt. Das gilt selbst dann, wenn der Eisenmangel (noch) nicht zur Anämie geführt hat.
\end{abstract}

Herz- und Niereninsuffizienz sind eng verknüpft und verstärken einander, sagte Prof. Roland Schaefer, Münster. Kommt dann noch ein Eisenmangel hinzu - wie dies bei den meisten chronischen, mit Entzündungsphänomenen einhergehenden Erkrankungen keine Seltenheit ist -, steigert dies die Morbidität und Mortalität.

Da die orale Eisensubstitution mit einer Resorptionsquote deutlich unter $10 \%$ wenig effektiv und überdies aufgrund der gastrointestinalen Nebenwirkungen mit einer schlechten Compliance behaftet ist, wird heute der intravenösen Applikation der Vorzug gegeben, insbesondere dann, wenn die Speicher rasch aufgefüllt werden sollen.

In der placebokontrollierten FAIR-HFStudie mit 459 Patienten war das Konzept der Eisensubstitution bei Herzinsuffizienz erprobt worden. Die 24-wöchige Behandlung mit Eisencarboxymaltose (Ferinject ${ }^{\circledR}$ ) führte sowohl zur subjektiven Besserung des Befindens im Patient Global Assessment Score als auch zur ärztlich festgestellten Besserung der NYHA-Klasse (Wahrscheinlichkeit für Besserung um mindestens eine Klasse: 2,4 im Vergleich zu Placebo). Patienten ohne Anämie profitieren in gleichem Maße wie jene mit Anämie.

Jetzt legte Studienleiter Prof. Stefan Anker, Charité Berlin, erstmals Resultate einer Post-hoc-Analyse vor, die zeigen, dass sich auch die Nierenfunktion unter der Substitution bessert. In der eisenbehandelten Gruppe nahm die glomeruläre Filtrationsrate um 3-4 $\mathrm{ml} / \mathrm{min}$ ab, während sie unter Placebo um o,6 $\mathrm{ml} / \mathrm{min}$ abnahm, und dies unabhängig vom Ausgangswert.

Über den Grund für diesen Benefit kann man derzeit nur spekulieren. Anker hält eine bessere Perfusion aller Organe, also auch der Nieren, für eine mögliche Erklärung sowie die bessere kardiale Funktion, die ebenfalls die renale Durchblutung steigert.

(ara) II

II Pressekonferenz beim Heart Failure Congress 2010, Berlin, 31. Mai 2010 (Veranstalter: Vifor) 